\title{
Verificação de rotulagem de dietas enterais industrializadas para pacientes diabéticos
}

\section{Verification of labeling of industrialized enteral diets for diabetic patients}

Resumo A rotulagem nutricional é definida como toda descrição destinada a informar o consumidor sobre as propriedades nutricionais de um alimento, compreendendo a declaração de valor energético e os principais nutrientes. A rotulagem de dietas enterais é uma ferramenta de extrema importância para a informação e segurança dos consumidores. Nutrição enteral especializada é indicada para pacientes com trato gastrointestinal funcionante, mas que requerem formulações especiais em virtude das doenças de base. A Terapia Nutricional Enteral (TNE) influi no controle da glicemia de pacientes diabéticos e a dieta efetivamente ofertada ao paciente pode ser avaliada como potencializadora de efeitos positivos ou negativos na glicemia. O objetivo deste estudo foi analisar a rotulagem nutricional de dietas enterais industrializadas para pacientes diabéticos em TNE no município de Fortaleza-CE. Foram analisados três rótulos de dietas enterais para diabéticos, de acordo com a legislação vigente no Brasil. Os rótulos avaliados apresentaram todas as informações previstas nos requisitos legais. Concluiu-se que as informações contidas nos rótulos das dietas enterais industrializadas, para pacientes diabéticos em TNE avaliadas, estão de acordo com os requisitos da legislação vigente.

Palavras-chave: Rotulagem nutricional. Terapia Nutricional. Diabetes Mellitus.

Abstract Nutritional labeling is defined as any description intended to inform the consumer about the nutritional properties of a food, including the declaration of energy value and the main nutrients. The labeling of enteral diets is an extremely important tool for consumer information and safety. Specialized enteral nutrition is indicated for patients with a functioning gastrointestinal tract, but who require special formulations due to underlying diseases. Enteral Nutritional Therapy (ENT) influences the control of glycemia in diabetic patients and the diet effectively offered to the patient can be evaluated as an enhancer of positive or ne-

GeruZa Maria Bevilaqua NOGUeira LiRA ${ }^{1}$

Luana Vieira Carvalho de OliveIRA $^{2}$

Joselito de Oliveira Neto ${ }^{3}$ Clarice Maria Araújo Chagas VERGARA ${ }^{1}$

${ }^{1}$ Universidade Estadual do Ceará (UECE), Fortaleza/CE-Brasil.

${ }^{2}$ Universidade de Fortaleza (UNIFOR), Fortaleza/CE-Brasil.

${ }^{3}$ Universidade Federal do Ceará (UFC), Fortaleza/CE-Brasil. gative effects on glycemia. The aim of this study was to analyze the nutritional labeling of industrialized enteral diets for diabetic patients in ENT in the city of Fortaleza-CE. Three enteral diet labels for diabetics were analyzed, according to the legislation in force in Brazil. The evaluated labels presented all the information provided for in the legal requirements. It was concluded that the information contained in the labels of the industrialized enteral diets, for diabetic patients in ENT assessed, are in accordance with the requirements of the current legislation.

Keywords: Nutritional Labeling. Nutrition Therapy. Diabetes MeLLITUS. 


\section{INTRODUÇÃO}

No Brasil, a legislação alimentícia iniciou-se na década de 1960 e, ao longo dos anos, vem sendo aprimorada com o propósito de melhorar a qualidade dos alimentos e de preservar a saúde da população. ${ }^{1}$ Com a criação e a regulamentação do Sistema Único de Saúde (SUS), boa parcela das questões relacionadas à produção e à comercialização de alimentos foi englobada pelo conjunto de ações da Vigilância Sanitária, sob a responsabilidade da Agência Nacional de Vigilância Sanitária (ANVISA), criada em 1999 e vinculada ao Ministério da Saúde, incluindo os aspectos referentes à rotulagem. ${ }^{2}$

Os rótulos são elementos de comunicação entre o produto e os consumidores, e devem auxiliá-los na decisão de compra, aumentando a eficiência do mercado e o bem- estar do consumidor. ${ }^{3}$ No entanto, o fato de a rotulagem ser obrigatória não significa que os consumidores a estejam utilizando como uma ferramenta para a escolha dos alimentos que devem compor sua dieta habitual e, assim, reduzir os excessos alimentares e, por seguinte, os danos ocasionados à saúde. ${ }^{4}$

A rotulagem nutricional é definida como toda a descrição destinada a informar o consumidor a respeito das propriedades nutricionais de um alimento, compreendendo a declaração de valor energético e os principais nutrientes. No entanto, é necessário que essas informações sejam compreendidas por todos aqueles que as utilizam. ${ }^{5,6,7}$ As informações fornecidas por meio da rotulagem contemplam um direito assegurado pelo Código de Defesa do Consumidor, o qual determina que a informação sobre produtos deve ser clara e com especificação correta de quan- tidade, composição e qualidade, bem como sobre os riscos que possam apresentar. ${ }^{8}$

No Brasil, a rotulagem nutricional é regulamentada pelas Resoluções de Diretoria Colegiada (RDCs) 360/03 e 359/03 da Agência Nacional de Vigilância Sanitária (ANVISA). Nesse sentido, devem ser declaradas, segundo a RDC 360/03, as quantidades por porção e a porcentagem do valor diário dos seguintes componentes: valor energético, teor de carboidratos, proteínas, gorduras totais, gorduras saturadas, gorduras trans, fibras alimentares e sódio. ${ }^{9,10}$ A RDC 359/03 estabelece as medidas e porções, incluindo a medida caseira e sua relação com a porção correspondente em gramas ou mililitros, detalhando também os utensílios utilizados com suas capacidades aproximadas. As porções indicadas nos rótulos de alimentos e bebidas foram determinadas com base em uma dieta de $2.000 \mathrm{kcal}$, considerando uma alimentação saudável. ${ }^{11,8}$ As dietas enterais devem atender às normas de rotulagem em geral e, também, às normas específicas RDC n. 449 , de 9 de setembro de 1999, pois o registro das dietas enterais analisadas é anterior à data de publicação da resolução $n$. 21, 13 de maio de 2015. Os estabelecimentos abrangidos por essa Resolução terão até 31 de maio de 2019 para promover as adequações necessárias, de acordo com o estabelecido a seguir: Redação dada pela Resolução - RDC no. 160, de 6 de junho de 2017. ${ }^{13}$

A rotulagem de dietas enterais é uma ferramenta importantíssima para a informação e segurança dos consumidores. A nutrição enteral (NE) consiste de alimentos formulados para fins especiais que possam ser utilizados por meio de sonda, podendo instilar os nutrientes diretamente no estômago ou 
no intestino, dependendo da condição do indivíduo. $\mathrm{O}$ suporte nutricional é uma importante terapêutica para pacientes que sofreram cirurgia no trato gastrintestinal ou apresentam distúrbios gastrintestinais graves, lesões do sistema nervoso central, depressão, câncer, entre outros. Atualmente, a NE é amplamente utilizada por ser segura, econômica e por preservar a imunidade e os mecanismos de defesa de pacientes. ${ }^{12}$

A NE especializada é indicada para pacientes com trato gastrintestinal funcionante, mas que requerem formulações especiais em virtude das doenças de base. Podem ter características poliméricas, oligomérica ou elementar, bem como serem nutricionalmente completas ou incompletas. ${ }^{13}$

É uma dieta pronta, balanceada, possuindo todos os nutrientes necessários. Pode ser apresentada sob a forma de pó, que deve ser preparada em água, ou sob a forma líquida pronta para ser administrada. As dietas enterais comercializadas se resumem a produtos prontos para serem servidos, com aporte nutricional balanceado, especialmente desenvolvido para atender necessidades ou restrições alimentares específicas. ${ }^{12}$

No Brasil, todas as etapas da TNE são regulamentadas por legislação específica, que estabelece requisitos mínimos para prescrição, formulação e administração da dieta e específicas atribuições para as instituições de saúde e para cada membro da equipe multidisciplinar (médico, enfermeiro, nutricionista e farmacêutico). Atualmente, dois tipos de sistemas para TNE estão disponíveis: sistema aberto (SA) e sistema fechado (SF). ${ }^{13}$

O SA se caracteriza por ser produzido em uma área restrita e específica, onde nu- trientes industrializados, em forma de pó ou líquida, são manipulados, seguindo as Boas Práticas de Manipulação, para que se atinja a composição desejada. O SA parece ter menores custos diretos, ainda que custos relacionados à implantação, manutenção e depreciação de área física e equipamentos, recursos humanos e validação de processos de manipulação devem ser considerados. ${ }^{14}$

O SF constitui-se de dietas líquidas, industrializadas, estéreis, acondicionadas em recipientes hermeticamente fechados e apropriados para conexão ao equipo de administração. Desse modo, a nutrição enteral em sistema aberto requer manipulação prévia à sua administração, enquanto o fechado não demanda áreas de preparo ou envase. ${ }^{13,14}$

Sabendo que a TNE é um fator que influi no controle da glicemia de pacientes diabéticos, a dieta efetivamente ofertada ao paciente pode ser avaliada como potencializadora de efeitos positivos ou negativos na glicemia. O Diabetes tipo 2 (DM) é considerado um problema mundial de saúde pública; cuja incidência e prevalência aumentam cada vez mais, alcançando proporções epidêmicas. Estima-se que cerca de 143 milhões de pessoas em todo o mundo sofram de DM e se acredita que esse número possa dobrar até 2030, quando o Brasil ocupará a sexta posição, com 11,3 milhões de casos. ${ }^{15}$

Configurando-se atualmente como uma epidemia resultante, em grande parte, do envelhecimento da população. Contudo, o sedentarismo, a alimentação inadequada e o aumento da obesidade também são responsáveis pela expansão global do DM. As hospitalizações atribuíveis ao DM repre- 
sentam 9\% dos gastos hospitalares do Sistema Único da Saúde. O DM está associado a complicações que comprometem a qualidade de vida e a sobrevida dos indivíduos. Além disso, acarreta elevados custos para o controle metabólico e o tratamento de suas complicações. ${ }^{16}$

Tendo em vista a importância da $\mathrm{Nu}-$ trição Enteral em reconstituir ou manter o estado nutricional do paciente, e a necessidade de se ofertar produtos com adequado padrão de qualidade, este estudo foi conduzido com o objetivo de verificar a rotulagem nutricional de dietas enterais industrializadas para pacientes diabéticos.

\section{MÉTOdos}

Trata-se de estudo descritivo e quantitativo. O estudo quantitativo preocupa-se com um nível de realidade que pode ser quantificado, mensurado. $\mathrm{O}$ estudo descritivo visa observar, registrar e descrever as características de determinada população ou situação em detalhe, estabelecendo correlações entre variáveis e definindo sua natureza. ${ }^{17}$

A pesquisa foi realizada nos meses de fevereiro e março de 2018 no município de Fortaleza-CE. O estudo consta de uma verificação da adequação da rotulagem das dietas enterais especializadas para diabetes embasada pela resolução n. 449 , de 9 de setembro de 1999, Resolução RDC n. 259, de 20 de setembro de 2002- ANVISA, RDC n ${ }^{\circ}$ 359 de 23 de dezembro de 2003 da ANVISA, RDC n. 360 de 23 de setembro de 2003 da ANVISA, Lei ${ }^{\circ} 10.674$, de 16 de maio de 2003, Resolução RDC n. 269 de 22 de setembro de 2005 , pois o registro das dietas enterais analisadas é anterior à data de publicação da resolução n. 21, de maio de 2015. Os estabelecimentos abrangidos por essa Resolução terão até 31 de maio de 2019 para promover as adequações necessárias, de acordo com o estabelecido a seguir: Redação dada pela Resolução - RDC n. 160, de 6 de junho de 2017. ${ }^{13}$

Foi realizada a verificação de elementos informativos e dos componentes nutricionais, de acordo com o que preconiza a legislação vigente. $\mathrm{Na}$ oportunidade foi realizada uma análise crítica dos elementos informativos e tabela nutricional entre eles os teores de carboidratos, proteína, gorduras totais, fibra, sódio $(\mathrm{Na})$, potássio $(\mathrm{K})$, cálcio $(\mathrm{Ca}) \mathrm{e}$ magnésio $(\mathrm{Mg})$ ofertados nessas dietas, com os valores de referência de ingestão diária, segundo RDC n. 21, de 13 de maio de 2015. ${ }^{13}$

Para a coleta dos rótulos de dietas enterais para diabéticos foi efetuado levantamento das empresas que produzem, no Brasil. Foram utilizados como critérios de inclusão: dietas enterais líquidas industrializadas, em sistema aberto, a indicação da dieta para diabético, além da produção e envase localizados no Brasil. Foi identificado um total de três amostras. Todas as amostras de dietas enterais para diabéticos foram adquiridas de acordo com o conteúdo disponível para venda de 1L, no comércio de Fortaleza-CE. As amostras foram codificadas por meio dos números (1, 2 e 3$)$ para facilitar a interpretação dos dados e manter a identidade das empresas em sigilo.

As conformidades e não conformidades das informações obrigatórias contidas nos rótulos das marcas selecionadas foram analisadas e estudadas, tomando como base a legislação vigente. Foi estabelecido um comparativo entre as marcas selecionadas. 
Os dados foram analisados com frequência simples em relação às conformidades e não conformidades dos rótulos e disponibilizados em tabelas. Foram analisados quanto aos princípios gerais de rotulagem, apresentação da informação nutricional e informações complementares e obrigatórias. A lista de verificação composta por 13 blocos com um total de 70 itens analisados, foi construída utilizando o programa Microsoft Office Word $^{\circledR}$ 2010. Foi preenchida com as denominações $(\mathrm{C})$ para o item conforme com a legislação e (NC) para o item não conforme com a legislação. Após a lista preenchida, procedeu-se à contagem de itens conformes e não conformes de cada rótulo analisado e utilizando a regra de três simples, onde o total de itens referentes à legislação representava $100 \%$ dos itens analisados e os itens conformes representavam o percentual de conformidade do rótulo perante a legislação.

Ancorado nesses dados foi elaborada uma tabela com percentual de itens em conformidade e itens não conformes de acordo com a Resolução RDC n. 449, de 9 de setembro de 1999 - dispõe sobre regulamento técnico de fórmulas para nutrição enteral. ${ }^{18}$ Resolução RDC n. 259, de 20 de setembro de 2002- ANVISA. Aprova o regulamento técnico sobre rotulagem de Alimentos Embalados. ${ }^{19}$ RDC no 359 de 23 de dezembro de 2003 da ANVISA, Regulamento Técnico de Porções de Alimentos Embalados Para Fins de Rotulagem Nutricional. ${ }^{11}$ RDC n. 360 de 23 de setembro de 2003 da ANVISA, Regulamento Técnico sobre Rotulagem Nutricional de Alimentos Embalados. ${ }^{9}$ Lei no 10.674 , de 16 de maio de 2003 obriga a que todos os produtos alimentícios comercializados informem sobrea presença de glúten. ${ }^{20} \mathrm{Re}$ solução RDC n. 269 de 22 de setembro de 2005, que aprova o Regulamento técnico sobre a Ingestão Diária Recomendada (IDR) de Fibras, Proteínas, Vitaminas e Minerais. Para uma análise de contribuição dos valores de sódio $(\mathrm{Na})$, potássio $(\mathrm{K})$, cálcio $(\mathrm{Ca})$ e magnésio $(\mathrm{Mg})$ com o que sugere Resolução n. 21 , de 13 de maio de $2015 .^{21,13}$

Os dados foram tabulados em planilha do programa Excel $^{\circledR}$ 2010, procedendo-se à estatística descritiva.

\section{Resultados E Discussão}

Os resultados a seguir foram obtidos a partir dos critérios metodológicos descritos para dietas enterais.

A Tabela 1 apresenta os resultados obtidos na verificação das informações obrigatórias contidas nos rótulos das amostras:

Conforme observado nos requisitos obrigatórios da Resolução de Diretoria Colegiada (RDC) 360/03, todos os rótulos contêm a denominação de venda do produto e uma lista de ingredientes precedida pela expressão “ingredientes” constando em ordem decrescente de concentração da sua composição final segundo determina a legislação. Sobre o requisito de identificação da origem do produto observa-se que os três rótulos atendem ao que determina a legislação indicando a Razão Social da marca do produto em seus dizeres de rotulagem, CNPJ, informações do importador, marca comercial, expressão "INDÚSTRIA BRASILEIRA", SAC, número do registro e o endereço completo do fabricante. 
Tabela 1: Verificação das informações obrigatórias contidas nos rótulos de dietas enterais para diabéticos. Fortaleza-CE, 2018.

\begin{tabular}{l|l|c|c}
\hline \multirow{2}{*}{ Disposição Normativa } & \multicolumn{1}{c|}{ Conformidade } & \multicolumn{2}{c}{ Embalagem } \\
\cline { 3 - 4 } & & \multicolumn{1}{c}{$\%$} & $\mathrm{n}$ \\
\hline RDC 449/1999 & Expressão de fórmulas para nutrição enteral & $100 \%$ & 3 \\
\hline RDC 259/2002 & Rotulagem de alimentos embalados & $100 \%$ & 3 \\
\hline RDC 360/2003 & $\begin{array}{l}\text { Apresentação no local de venda } \\
\text { Existência de informações obrigatórias } \\
\text { Concordância entre valor energético apresentado } \\
\text { e calculado }\end{array}$ & $100 \%$ & 3 \\
\hline RDC 359/2003 & Conteúdo líquido & $100 \%$ & 3 \\
\hline LEI 10.674/2003 & Expressão quanto à presença de glúten & $100 \%$ & 3 \\
\hline RDC 269/2005 & Ingestão diária recomendada (IDR) & $100 \%$ & 3 \\
\hline
\end{tabular}

Fonte: Elaborado pelos autores.

Os rótulos 1,2 e 3 , atendem ao que se preconiza, pois, os mesmos apesar de indicar os municípios de origem de fabricação, utilizam expressões que identificam o país de origem. A identificação de origem permite que o consumidor saiba quem é o fabricante do produto e onde ele foi fabricado. São informações importantes para o consumidor saber qual a procedência do produto e entrar em contato com o fabricante, se for necessário?.

Os requisitos gerais de rotulagem estipulados pela Resolução RDC n. 259 de 20 de setembro de 2002 foram verificados e os rótulos analisados. Um total de 100\% estava em acordo com o exigido pela legislação.

Dados referentes à lista de ingredientes, bem como declaração de aditivos utilizados, foram encontrados nos rótulos analisados. Segundo a legislação RDC n. 449/1999 os aditivos alimentares devem ser declarados fazendo parte da lista de ingredientes e precisa constar a função principal ou fundamental do aditivo no alimento e seu nome completo ou seu número INS (Sistema Internacional de Numeração) ou ambos. ${ }^{18}$

No que se refere a conteúdo líquido, todas as marcas estão em conformidade com a legislação. O conteúdo líquido possui mais de $4 \mathrm{~mm}$, encontra-se com cor contrastante, não confundindo com a cor da embalagem. Segundo a RDC n. 359/03, o conteúdo líquido precisa constar na parte principal da embalagem e deve ser de cor contrastante com o fundo onde estiver impresso, de modo a transmitir ao consumidor uma fácil, fiel e satisfatória quantidade comercializada. ${ }^{11}$

As três marcas de dietas enterais apresentaram declaração da densidade energética do produto, expressa em $\mathrm{kcal} / \mathrm{mL}$ no painel principal RDC n. 449 , de 9 de setembro de 1999. Segundo a RDC n. 21, de 13 de maio de 2015 as marcas exibiram osmolaridade do produto pronto para o consumo; instruções de preparo que assegurem homogeneização adequada para administração via tubo; instrução de administração do 
produto; informação relacionada às precauções de uso; instrução de conservação do produto, inclusive após abertura da embalagem; advertência em destaque e negrito: "Proibido o uso por via parenteral"; advertência em destaque e negrito: "Usar somente sob orientação médica ou de nutricionista"; mostrando que as três marcas estão de acordo com a nova legislação.

Todas as marcas apresentaram a inscrição "não contém Glúten" em seus rótulos conforme a Lei n. 10.674, de 16 de maio de 2003 , que obriga a que os produtos alimentícios comercializados informem sobre a presença de glúten, como medida preventiva e de controle da doença celíaca. A única terapia nutricional para essa patologia é a restrição total de alimentos que contenham glúten. ${ }^{22}$ Sendo assim torna-se importante intensificar a questão da confiabilidade das informações que se relacionam diretamente à segurança dos alimentos, criando um elo entre o consumidor, o alimento e o produtor. Portanto, o acesso a essas informações corretas é de suma importância para a prevenção do risco de alergias alimentares.

Todos os rótulos avaliados apresentaram informações nutricionais agrupadas em um mesmo local em forma de tabela vertical, aprovado pela Resolução RDC n. 360 de 23 de dezembro de 2003. ${ }^{9}$ Foi observada a adequação de rótulos de dietas enterais quanto à forma de expressão da informação nutricional, em idioma legível e visível, declaração do valor energético e nutrientes obrigatórios.

A qualidade nutricional da fórmula enteral é um fator importante para a diminuição da morbidade e mortalidade de pacientes. Além da qualidade dos ingredientes, o conhecimento da adequação e distribuição dos nutrientes nos formulados industrializados, somados à garantia de que o pacien- te receba o que lhe foi prescrito, são fatores que garantem um bom aproveitamento desse tipo de alimentação. ${ }^{23,14}$

A resolução 21, de 13 de maio de 2015 determina para dietas enterais os valores máximos e mínimos permitidos para cada mineral indicado: sódio (29 - $115 \mathrm{mg})$, potássio (80 - $327 \mathrm{mg})$, magnésio (13 - 34 $\mathrm{mg})$, ferro $(0,7-2,3 \mathrm{mg})$ e cálcio $(50-125$ $\mathrm{mg}$ ) para cada $100 \mathrm{kcal}$ de dieta enteral. ${ }^{13}$ Portanto, foi possível realizar neste estudo uma verificação no rótulo dos valores mínimos e máximo de cada mineral e compará-los com a legislação. Entretanto, observa-se que a maioria estava adequada entre seus componentes. Apenas a amostra 03 exibiu o sódio acima do preconizado pela legislação onde o limite máximo recomendado seria $115 \mathrm{mg}$ e o encontrado na amostra foi 150mg. Como apresenta a Tabela 2:

O consumo de macro e micronutrientes é fundamental para manter as necessidades energéticas do organismo e suas reservas nos tecidos e músculos. Quando não atingido, resulta em desnutrição e quando ocorrem excessos pode também ser prejudicial por sobrecarregar o organismo. ${ }^{24} \mathrm{O}$ sódio é um íon muito importante na manutenção da hidratação, a redução no consumo está diretamente relacionada à prevenção de doenças crônicas. Embora presente naturalmente em diversos alimentos, em quantidades que atendem às recomendações humanas, a maior parte do sódio da dieta é proveniente dos compostos sódicos adicionados no processamento dos alimentos ou, em menor escala, do sal de mesa. Sabe-se que o cloreto de sódio, quando ingerido em quantidades superiores às necessidades do organismo, está associado a maior incidência de hipertensão arterial na população. ${ }^{25}$ 
Tabela 2: Verificação das informações nutricionais contidas nos rótulos. Fortaleza-CE, 2018

\begin{tabular}{|c|c|c|c|c|c|c|}
\hline & \multicolumn{2}{|l|}{ Amostra 01} & \multicolumn{2}{|l|}{ Amostra 02} & \multicolumn{2}{|l|}{ Amostra 03} \\
\hline & \multicolumn{2}{|c|}{ Porção por $100 \mathrm{ml}$} & \multicolumn{2}{|c|}{ Porção por $100 \mathrm{ml}$} & \multicolumn{2}{|c|}{ Porção por $100 \mathrm{ml}$} \\
\hline & $\begin{array}{l}\text { Quantidade por } \\
\text { porção }\end{array}$ & $\% \mathrm{VD}$ & $\begin{array}{c}\text { Quantidade por } \\
\text { porção }\end{array}$ & $\% \mathrm{VD}$ & $\begin{array}{l}\text { Quantidade por } \\
\text { porção }\end{array}$ & $\% \mathrm{VD}$ \\
\hline Valor energético & $100 \mathrm{kcal}=417 \mathrm{KJ}$ & ** & $100 \mathrm{kcal}=420 \mathrm{KJ}$ & ** & $\begin{array}{c}112 \mathrm{kcal}=469 \\
\mathrm{KJ}\end{array}$ & ** \\
\hline Carboidratos & $8,1 \mathrm{~g}$ & $* *$ & $9,8 \mathrm{~g}$ & $* *$ & $9,5 \mathrm{~g}$ & $* *$ \\
\hline Proteínas & $4,2 \mathrm{~g}$ & $* *$ & $3,9 \mathrm{~g}$ & $* *$ & $4,9 \mathrm{~g}$ & $* *$ \\
\hline Gorduras totais & $5,4 \mathrm{~g}$ & $* *$ & $5,1 \mathrm{~g}$ & ** & $6,0 \mathrm{~g}$ & ** \\
\hline Fibra alimentar & $1,4 \mathrm{~g}$ & $* *$ & $1,6 \mathrm{~g}$ & $* *$ & $1,5 \mathrm{~g}$ & $* *$ \\
\hline Sódio & $93 \mathrm{mg}$ & 4 & $94 \mathrm{mg}$ & 4 & $150 \mathrm{mg}$ & 6 \\
\hline Potássio & $130 \mathrm{mg}$ & $* *$ & $156 \mathrm{mg}$ & $* *$ & $140 \mathrm{mg}$ & $* *$ \\
\hline Cálcio & $70 \mathrm{mg}$ & 7 & $110 \mathrm{mg}$ & 11 & $90 \mathrm{mg}$ & 9 \\
\hline Magnésio & $20 \mathrm{mg}$ & 8 & $28 \mathrm{mg}$ & 11 & $17 \mathrm{mg}$ & 7 \\
\hline Ferro & $1,3 \mathrm{mg}$ & 9 & $1,5 \mathrm{mg}$ & 11 & $1,6 \mathrm{mg}$ & 11 \\
\hline
\end{tabular}

Fonte: Elaborado pelos autores.

As fibras possuem muita importância tanto em caso de constipação quanto em ocasiões de diarreia. Também têm efeito benéfico na manutenção da mucosa, no perfil lipídico e da glicose, e na manutenção da imunidade. Para tanto, é necessário que seja analisado individualmente cada caso a fim de agir da melhor forma para cada caso específico. A dieta com fibras protege a motilidade intestinal, prevenindo complicações ou distúrbios. ${ }^{26,27}$

Diversos autores já relataram problemas de não conformidade quanto à rotulagem de alimentos no Brasil e apontaram algumas lacunas existentes na legislação relacionada. O rótulo faz parte da embalagem e por mais elaborado ou simples que ele seja deve conter obrigatoriamente certas informações do produto. Ele pode desempenhar a função de identificação, de produto ou marca; de clas- sificação; de descrição, fabricante, data de fabricação, conteúdo e como ser usado com segurança; de promoção do produto por meio da aparência gráfica atraente. ${ }^{28}$

Estudos analisados concluem que, em alimentos de uma forma geral, tanto importados e como nacionais, as inadequações são as mesmas: ausência de informações sobre o número do lote, a data de fabricação, o prazo de validade, especificação dos corantes adicionados intencionalmente e informação sobre presença de glúten. ${ }^{1}$

Nenhuma irregularidade foi encontrada nos rótulos dos produtos enquadrados nas Portarias n. 34/98 (alimentos de transição para lactentes e crianças de primeira infância) 4 rótulos, 977/98 (fórmulas infantis para lactentes) 6 rótulos e 223/98 (complementos alimentares para gestantes e nutrizes) 1 rótulo. ${ }^{29}$ Evidenciaram a necessidade 
de fiscalização e revisão da legislação de rotulagem nutricional no Brasil a fim de uniformizar as porções declaradas nos rótulos. ${ }^{30}$ Amostras foram coletadas entre novembro de 2006 e fevereiro de 2007 e avaliaram a rotulagem nutricional de alimentos com alto teor de ácidos graxos trans. Foi observado que a maior parte dos produtos analisados $(55,3 \%)$ ainda não havia se adequado à $\mathrm{RDC} 360 / 03$. Segundo essa norma os produtos deveriam estar adequadamente rotulados até 31 de julho de 2006. ${ }^{31}$

A declaração nutricional apresentou-se no estudo como um potencial instrumento de educação nutricional, visto que os consumidores reconheceram a importância e a necessidade da declaração nutricional nos rótulos dos alimentos. ${ }^{7}$ Os dados mostraram que os consumidores tinham uma compreensão parcial sobre os dizeres de rotulagem veiculados na declaração nutricional e identificaram alguns ruídos na comunicação entre o produto e o consumidor, incluindo a legibilidade e a aptidão para interpretar as informações nutricionais. Esses achados são semelhantes aos de outros estudos realizados nos Estados Unidos, que revelam que muitos consumidores ainda têm dificuldade de entender e aplicar a informação nutricional na sua vida, mesmo entre profissionais qualificados. Para muitos entrevistados, a declaração nutricional serviu para subsidiar o controle dietoterápico para determinados tipos de doenças crônicas não transmissíveis. $\mathrm{O}$ mesmo foi relatado em outro estudo, no qual pessoas com história de doença crônica referiram que verificavam e utilizavam a rotulagem nutricional. ${ }^{32}$

No ato da compra dos alimentos, $43 \%$ dos consumidores brasileiros, buscam nas embalagens informações sobre benefícios para a saúde. ${ }^{33}$ Em recentes estudos, concluiu-se que $61 \%$ dos frequentadores de supermercados leem os rótulos dos produtos que compram, porém, a autora ressalta que tal conduta se refere, particularmente, àqueles consumidores com problemas de saúde e/ou de classe sociais mais elevadas. ${ }^{34}$ No entanto, vários estudos identificam a dificuldade do consumidor em compreender tais informações por não serem disponibilizadas de forma clara e em linguagem acessível. Além disso, muitos consumidores não conhecem a rotulagem nutricional, e alguns não demonstram interesse por esses dados. ${ }^{34,1}$

\section{CONSIDERAÇõeS FINAIS}

Os resultados obtidos demonstraram que todas as amostras estavam de acordo com as normas vigentes para rotulagem do segmento no Brasil. Quando comparado à nova legislação apenas uma amostra apresentou desacordo com a norma para rotulagem de alimentos. A amostra exibiu o sódio acima do preconizado pela legislação. Sabe-se que o cloreto de sódio, quando ingerido em quantidades superiores às necessidades do organismo, está associado a maior incidência de hipertensão arterial na população.

A discriminação das informações gerais do alimento, bem como a rotulagem nutricional são benéficas tanto para os consumidores como para as empresas. Consumidores especiais como portadores de diabetes bem informados consomem os alimentos com maior segurança não só em relação ao valor calórico e demais nutrientes do produto, mas também em relação aos consti- 
tuintes que podem desencadear reações diversas no organismo, e as empresas ganham em credibilidade, adquirindo a confiança dos consumidores.

\section{AGRADECIMENTOS}

À Universidade Estadual do Ceará pelo apoio e incentivo à pesquisa.

\section{REFERÊNCIAS}

1. Mira I., Carvalho F., Celso D. Avaliação de conformidade da rotulagem de queijos comercializados na região metropolitana de Salvador, BA. Hig. aliment. 2017; 31: 151-6.

2. Ferreira $\mathrm{AB}$, Lanfer-marquez UM. Legislação brasileira referente à rotulagem nutricional de alimentos. Rev. Nutr., Campinas. 2007; 20 (1): 83-93.

3. Teixeira SM., Chiconatto P., Mazur CE., Schmitt V. Alimentos consumidos por crianças em idade escolar: análise das tabelas nutricionais. Revista Brasileira de Obesidade, Nutrição e Emagrecimento. São Paulo. jan./dez. 2017; 11 (67): 531- 40.

4. Cavada GS., Paiva FF., Helbig E., Borges LR. Rotulagem nutricional: você sabe o que está comendo? Revista Brasileira de Tecnologia de Alimentos. Campinas. mai. 2012; 15 (spe): 84-8.

5. AGÊNCIA NACIONAL DE VIGILÂNCIA SANITÁRIA; UNIVERSIDADE DE BRASÍLIA. Rotulagem nutricional obrigatória: manual de orientação às indústrias de alimentos. 2. ed. rev. Brasília, 2005. $44 \mathrm{p}$.

6. Câmara CCM, Marinho CLC, Guilam RCM. Análise crítica da rotulagem de alimentos diet e light no Brasil. Caderno de Saúde Coletiva. Rio de Janeiro. 2008; 16 (1): 35-52.

7. Souza SMFC, Lima KC, Miranda HF, Cavalcanti FID. Utilização da informação nutricional de rótulos por consumidores de Natal, Brasil. Revista Panamericana de Salud Pública. Washington. $2011 ; 29$ (5): 337-43.

8. Araújo WDR. Importância, estrutura e legislação da rotulagem geral e nutricional de alimentos industrializados no Brasil. Revista Acadêmica Conecta. 2017; 2 (1): 35-50.

9. Ministério da Saúde. Agência Nacional de Vigilância Sanitária. Resolução nº. 360, de 23 de dezembro de 2003b. Aprova o Regulamento Técnico sobre Rotulagem Nutricional de Alimentos Embalados, tornando obrigatória a rotulagem nutricional. Diário Oficial da União, Brasília, 2003.

10. Lobanco CM., Vedovato GM., Cano CB., Bastos DHM. Fidedignidade de rótulos de alimentos comercializados no município de São Paulo, SP. Revista de Saúde Pública. São Paulo. 2009; 43 (3): 499-505.

11. Ministério da Saúde. Agência Nacional de Vigilância Sanitária. Resolução no ${ }^{\mathbf{0}}$. 359, de 23 de dezembro de 2003a. Aprova o Regulamento Técnico de Porções de Alimentos Embalados Para Fins de Rotulagem Nutricional. Diário Oficial da União, Brasília, 2003.

12. Rocha ADJSC., Oliveira ATV., Cabral NAL., Gomes RDS., Guimarães TA., Rodrigues WB., Silva E LDS. Causas de interrupção de nutrição enteral em unidades de terapia intensiva. Rev. Pesq. Saúde. Maranhão. jan./abr. 2017; 18 (1): 49-53.

13. BRASIL. Ministério da Saúde. Regulamento técnico de fórmulas para nutrição enteral. Resolução RDC no. 21, de 13 de maio de 2015. Diário Oficial da República Federativa do Brasil, Poder Executivo, Brasília, DF, 13 mai. 2015.

14. Sperafico BM, Rabito IE, Michielin BF. Relação entre o uso de terapia nutricional enteral e o controle glicêmico em pacientes críticos. Nutr. clín. diet. hosp. Curitiba. 2016; 36 (4): 73-81.

15. Silva AMDOE., Andrade-wartha ERSD., Carvalho EBTD., Lima A., Novoa AV., Mancini-filho J. Efeito do extrato aquoso de alecrim (Rosmarinus officinalis L.) sobre o estresse oxidativo em ratos diabéticos. Rev. Nutr. Campinas. 2011; 24 (1): 121-30. 
16. Schmidt MI., Bruce BD., Hoffmann JF., Moura L., Malta DC., Carvalho RMSV. Prevalência de diabetes e hipertensão no Brasil baseada em inquérito de morbidade auto-referida, Brasil, 2006. Rev. Saúde Pública. São Paulo. nov. 2009; 43 (2): 74-82.

17. Bordalo AA. Estudo transversal e/ou longitudinal. Rev. Para. Med. Belém. 2006; 20(4): 5.

18. BRASIL. Ministério da Saúde. Regulamento técnico de fórmulas para nutrição enteral. Resolução RDC no. 449, de 9 de setembro de 1999. Diário Oficial da República Federativa do Brasil, Poder Executivo, Brasília, DF, 10 set. 1999.

19. Ministério da Saúde. Agência Nacional de Vigilância Sanitária. Resolução no . 259, de 20 de setembro de 2002. Aprova o Regulamento Técnico sobre Rotulagem de Alimentos Embalados. Diário Oficial da União, Brasília, 2002.

20. Lei $\mathbf{n}^{\mathbf{0}} \mathbf{. 1 0 . 6 7 4}$, de maio de 2003c. Obriga a que os produtos alimentícios comercializados informem sobre a presença de glúten, como medida preventiva e de controle da doença celíaca. Diário Oficial da União, Brasília, DF, 19 de mai. 2003. Seção 1.

21. BRASIL. Ministério da Saúde. Agência Nacional de Vigilância Sanitária. Resolução RDC no . 269, de 22 de setembro de 2005. Aprova o Regulamento Técnico sobre a Ingestão Diária Recomendada (IDR) de Proteína, Vitaminas e Minerais. Diário Oficial da União, Brasília, 2005.

22. Ribeiro ROR., Cunha FL., Carneiro CS., Mársico ET. Avaliação da adequação da rotulagem de geleias reais. Revista Brasileira de Ciência Veterinária. Rio de Janeiro. mai/ago. 2012. 19 (2): 94-97.

23. Isidro MF, Lima DSC. Adequação calórico-proteica da terapia nutricional enteral em pacientes cirúrgicos. Revista Associação Médica Brasileira, São Paulo. set./out. 2012.58 (5): 580-86.

24. DITEN-PROJETO DIRETRIZES. Recomendações Nutricionais para adultos em terapia nutricional enteral e parenteral. Sociedade Brasileira de Nutrição Parenteral e Enteral. Sociedade Brasileira de Clínica Médica. Associação Brasileira de Nutrologia. 2011.1 (1): 1-10.

25. Webster JL., Dunford EK., Hawkes C., Neal BC. Salt reduction initiatives around the world. J. Hypertens. Utah. 2011. 29 (6): 1043-50.

26. Castro RCB. Constipação é mais frequente do que diarreia em pacientes alimentados por nutrição enteral. Nutritotal. São Paulo. 2012. 27 (4): 533-920.

27. Magnoni D. Fibras na nutrição enteral. IMeN - Instituto de Metabolismo e Nutrição. São Paulo. 2015. 18 (1): 3-26.

28. Araújo WDR. Importância, estrutura e legislação da rotulagem geral e nutricional de alimentos industrializados no Brasil. Revista Acadêmica Conecta. 2017. 2 (1): 35-50.

29. Yoshizawa N., Pospissil RT., Valentim AG., Seixas D., Alves FS., Cassou F., Yoshida I., Sega RA., Cândido LMB. Rotulagem de alimentos como veículo de informação ao consumidor: adequações e irregularidades. B.CEPPA. Curitiba. jan./jun. 2003. 21(1): 169-180.

30. Kliemann N., Veiros MB., Gonzalez-chica D., Proenca RPC. Porções de referência para a população brasileira: uma análise dos rótulos de alimentos processados. Rev. Nutr. Campinas. 2014 27 (3): 329- 341.

31. Dias JR, Goncalves ÉCBA. Avaliação do consumo e análise da rotulagem nutricional de alimentos com alto teor de ácidos graxos trans. Ciênc. Tecnol. Aliment. Campinas. 2009. 29 (1): 177- 182.

32. Burton S., Creyer EH., Kees J. Huggins K. Attacking the obesity epidemic: the potential health benefits of providing nutrition information in restaurants. Am. J. Public Health. EUA. set. 2006. 96 (9): 1669-1675.

33. Coutinho JG. Estabelecimento de alegação de saúde nos rótulos de alimentos e bebidas embalados. Brasília, 2004. 105f. Dissertação (Mestrado em Nutrição Humana) - Universidade de Brasília, Brasília, 2004. 
34. MARINS, B. R. Análise do hábito de leitura/recepção das informações contidas em rótulos de produtos alimentícios embalados, pela população adulta frequentadora de supermercados, no Município de Niterói/RJ. 2004. 130 f. Dissertação (Mestrado em Vigilância Sanitária) - Fundação Oswaldo Cruz, Instituto Nacional de Controle de Qualidade em Saúde, Rio de Janeiro, 2004.

\section{DADOS DOS AUTORES}

\section{Geruza Maria Bevilaqua Nogueira Lira}

Nutricionista - Universidade de Fortaleza (UNIFOR). Especialização em Vigilância Sanitária de Alimentos - Universidade Estadual do Ceará (UECE). geruza.bevilaqua@yahoo.com.br

\section{Luana Vieira Carvalho de Oliveira}

Nutricionista - Universidade de Fortaleza (UNIFOR). Especialização em Vigilância Sanitária de Alimentos - Universidade Estadual do Ceará (UECE).

\section{Joselito de Oliveira Neto}

Fisioterapeuta - Universidade de Fortaleza (UNIFOR). Doutorando em Biotecnologia - Universidade Federal do Ceará (UFC). joselitoneto@yahoo.com.br

\section{Clarice Maria Araújo Chagas Vergara}

Professora Adjunta e Coordenadora do Curso de Nutrição da Universidade Estadual do Ceará (UECE). Pós-doutorado em Saúde Coletiva - Universidade Estadual do Ceará (UECE). clarice.araujo@uece.br

Submetido em: 2-4-2020

Aceito em: 24-9-2020 